FORMATION Formation emploi

Revue française de sciences sociales

93 | janvier-mars 2006

Au-delà du genre, l'insertion

\title{
La ségrégation des hommes et des femmes dans les métiers : entre héritage scolaire et construction sur le marché du travail
}

The occupational segregation of men and women: a heritage resulting from educational segregation combined with labour market practices

Geschlechtsspezifische Segregation der Arbeitswelt: schulisches Erbe oder berufliches Konstrukt?

Thomas Couppié et Dominique Epiphane

\section{OpenEdition \\ Journals}

Édition électronique

URL : http://journals.openedition.org/formationemploi/2204

ISSN : 2107-0946

Éditeur

La Documentation française

Édition imprimée

Date de publication : 1 mars 2006

Pagination : 11-27

ISSN : 0759-6340

Référence électronique

Thomas Couppié et Dominique Epiphane, «La ségrégation des hommes et des femmes dans les métiers : entre héritage scolaire et construction sur le marché du travail », Formation emploi [En ligne], 93 | janvier-mars 2006, mis en ligne le 24 février 2010, consulté le 30 octobre 2020. URL : http:// journals.openedition.org/formationemploi/2204 


\section{La ségrégation des hommes et des femmes dans les métiers : entre héritage scolaire et construction sur le marché du travail}

par Thomas Couppié et Dominique Epiphane*

Les différences entre sexes sur le marché du travail ne reproduisent pas mécaniquement les différences scolaires. Une ségrégation professionnelle peut suivre, renforcer ou compenser la ségrégation éducative.

La question sociale de l'égalité professionnelle entre hommes et femmes n'a cessé de grandir au cours de ces dernières années. Parmi la multiplicité des sources d'inégalités existantes, les études pointent, de façon récurrente, le handicap que constitue la concentration des emplois féminins dans un nombre restreint de professions et la difficulté qu'éprouvent les femmes à accéder aux positions d'emploi les plus prestigieuses (Maruani, 2000). En réponse à ces constats, les pouvoirs publics ont largement privilégié, comme outil d'intervention, les politiques publiques éducatives (Torsat 1999). Dans ce cadre d'action, la diversification des orientations féminines est donc vue comme un instrument de lutte pertinent et efficace contre la concentration professionnelle des femmes. Ce sont précisément les potentialités et les limites de cet instrument que nous nous proposons d'interroger dans cet article.

Poser le problème de la concentration de l'emploi féminin en termes d'orientation scolaire revient à
* Thomas Couppié est démographe, Dominique Epiphane, sociologue. Chargés d'études au Céreq, au Département des entrées dans la vie active, ils travaillent ensemble sur les disparités d'insertion entre hommes et femmes et sur les questions de ségrégation professionnelle. Dominique Epiphane a travaillé également sur les cadres et sur les diplômés de l'enseignement supérieur. Elle a publié "La féminisation de la catégorie cadres au miroir de l'insertion des jeunes », in Quand les jeunes entrent dans I'emploi, Arliaud M., Eckert H. (dir), Éditions La Dispute, 2002 et "Hommes-femmes: les inégalités demeurent », Alternatives économiques, Horssérie "Pratique » n 17, 2005. Thomas Couppié a aussi travaillé sur la correspondance emploi-formation dans le tertiaire et l'insertion comparée des jeunes en Europe. II a publié avec Michèle Mansuy, "L'insertion 
professionnelle des débutants en Europe : des situations contrastées "Économie \& Statistique n³78-379, 2004 et avec Alberto Lopez et Jean-François Giret « Des formations initiales aux premiers emplois: une correspondance plutôt mal assurée », in Des formations pour quels emplois ?, Giret J.-F., Lopez A., Rose J. (dir.), Éditions La Découverte, 2005.

privilégier une approche longitudinale dans l'analyse de la construction de cette concentration. Dans cette perspective, le positionnement des jeunes hommes et des jeunes femmes dans les différents segments professionnels, à leur arrivée sur le marché du travail, s'est déjà dessiné en partie en amont, sur les bancs de l'école, ceux-ci et celles-là n'ayant pas accédé aux mêmes espaces d'éducation. C'est donc bien dans le temps que s'élaborent les différences de parcours masculins et féminins, et l'étude comparative de l'entrée dans la vie active des jeunes hommes et des jeunes femmes suppose d'appréhender les différents moments où se jouent les « destins sexués » des unes et des autres.

La ségrégation que l'on observe entre les hommes et les femmes sur le marché du travail est l'aboutissement d'un processus long. Elle commence en effet dès la petite enfance, s'enracine sur les bancs de l'école, du lycée et de l'université pour se cristalliser à l'arrivée sur le marché du travail, moment où les individus choisissent et sont choisis par les employeurs. La ségrégation professionnelle est donc le fruit, longuement mûri, d'une construction sociale qui inclut mais dépasse largement le phénomène, plus ponctuel, de discrimination sur le marché du travail au sens où l'entendent les juristes (inégalité de traitement de personnes égales (Kessler, 1997)) et les économistes (résidu inexpliqué par les caractéristiques individuelles (Silvera, 1996)). Sans nier l'existence de pratiques discriminatoires du côté de la demande de travail, c'est-à-dire des employeurs, la ségrégation professionnelle s'élabore plus profondément, plus insidieusement et d'abord, du côté de l'offre de travail, c'est-à-dire des jeunes. Puis, prenant appui sur la ségrégation éducative, la ségrégation professionnelle se construit en aval sur le marché du travail. Nous parlerons ici de "ségrégation» comme une «séparation de fait», sans nous prononcer a priori sur ce qui relève des contraintes et déterminismes sociaux (les " structures structurées ») qui pèsent sur les agents et qui placent les filles et les garçons, les hommes et les femmes à des positions particulières de l'espace social... et sur ce qui relève des stratégies que déploient les acteurs pour se placer au mieux sur le marché du travail en fonction des possibilités et des contraintes objectives qui les attendent.

\section{Le poids du passé en question}

La progression spectaculaire des scolarités féminines n'a pas ébranlé les lois de la reproduction sociale, puisque les filles continuent, grosso modo, à s'orienter vers les mêmes filières que les générations précédentes. L'expansion de ces scolarités s'est accomplie dans le «strict respect de l'ordre scolaire établi : aucun bouleversement ni dans le régime de la méritocratie, ni dans le mécanisme de l'hérédité sociale, ni même enfin dans les ségrégations sexuelles des différentes filières. » (Establet 1988). Depuis les années 90, les sociologues de l'éducation n'ont cessé de souligner ce double phénomène d'augmentation du capital scolaire des jeunes filles et de maintien d'une forte ségrégation au sein des filières $\mathrm{du}$ système scolaire, à tous les niveaux de formation (Duru-Bellat, 1990; Duru-Bellat et al., 2001 ; Baudelot et Establet, 1992 ; Terrail, 1992). Quel que soit le poids que les uns et les autres accordent aux déterminismes sociaux et la manière dont ils expliquent les comportements et les choix scolaires sexuellement différenciés, tous s'accordent sur le fait que la progression extraordinaire des jeunes filles dans l'institution scolaire au cours du siècle dernier ne s'est pas traduite par une généralisation de la mixité des filières, cette situation ne facilitant ainsi guère l'accession des femmes aux mêmes espaces professionnels que les hommes.

Dans le champ de l'insertion professionnelle des jeunes, le maintien des jeunes filles dans des segments particuliers du système scolaire va apparaître comme un facteur central, explicatif d'une (bonne) part des différences observées sur le marché du travail entre les jeunes hommes et les jeunes femmes (Couppié, Epiphane et Fournier, 1997 ; Couppié et Epiphane, 2001). Les explications traditionnellement avancées pour interpréter les disparités professionnelles des jeunes hommes et femmes reposent alors essentiellement sur une hypothèse implicite de reproduction dans le système productif de la ségrégation opérée en 
amont, dans le système scolaire. Ne suivant pas les mêmes formations, les jeunes femmes occuperaient logiquement des professions différentes. Les mécanismes d'allocation des individus dans les différents groupes professionnels prolongeraient donc, mécaniquement, la ségrégation éducative induite par les orientations scolaires sexuellement clivées.

À l'instar des travaux qui ont pointé que les variations des destinées sociales selon le milieu d'origine ne se forgent pas seulement à l'école mais aussi au long de la vie professionnelle (Goux et Maurin, 1997 ; Vallet, 1999), l'ambition de notre travail est de mieux appréhender la divergence des positions professionnelles occupées par les jeunes hommes et les jeunes femmes, une fois prises en compte les caractéristiques scolaires propres à chacun des deux sexes. Il apparaîtra que la ségrégation professionnelle entre hommes et femmes sur le marché du travail n'est pas un simple héritage de la ségrégation éducative. En effet, les mécanismes de distinction conduisant à séparer les filles des garçons dans les emplois empruntent des chemins plus complexes - et plus longs - que les seuls chemins dessinés par les parcours scolaires. Il s'agira aussi d'élaborer des indicateurs capables de caractériser l'éventuelle hétérogénéité entre les groupes professionnels.

\section{Un passé (dé)composé au présent}

Notre objectif est d'analyser le processus qui conduit à la ségrégation professionnelle entre hommes et femmes. Nous postulons alors que ce processus se construit en deux étapes distinctes, articulées autour de la sortie de formation initiale. Dans la première, des disparités d'orientations naissent entre les filles et les garçons dans les formations (ségrégation éducative). Dans la seconde, apparaissent des répartitions différenciées des filles et des garçons dans les professions : pour partie initiées par les orientations scolaires antérieures, ces répartitions sont aussi infléchies par des mécanismes d'appariement - entre individus et emplois - sexuellement différenciés et prenant corps sur le marché du travail (ségrégation professionnelle).

La décomposition de cette seconde étape va alors permettre de distinguer les différentes sources de la ségrégation professionnelle. La première s'alimente, plus ou moins parfaitement, des disparités d'orientations entre les filles et les garçons dans les formations ; elle contribue ainsi à la répartition différenciée des jeunes hommes et des jeunes femmes dans les professions. Cette ségrégation "professionnelle d'origine éducative» (qu'on peut noter SPeduc) est l'héritière directe de la ségrégation éducative (qu'on peut noter SE). Cependant, cette transmission n'a rien d'automatique. La «qualité » de la transmission dépend en effet de la nature des relations entre formations et emplois. Ainsi, la ségrégation réalisée en formation ne sera transmise que si les jeunes sortant d'une formation dominée par un des deux genres vont vers des groupes professionnels dominés eux aussi par ce genre-là. En renversant le point de vue, plus les groupes professionnels font appel à des formations de même genre, mieux sera conservée/transmise la ségrégation éducative. Au contraire, les groupes professionnels mobilisant des individus issus de formations de genre opposé « cassent » ces processus de transmission ou « effacent » la ségrégation éducative. Ils pratiquent alors une forme de déségrégation qui passe par le rapprochement d'individus séparés au moment de leurs études.

La deuxième source de ségrégation professionnelle n'est pas liée au parcours de formation. Elle se construit sur le marché du travail par le jeu d'allocations différenciées dans les divers groupes professionnels des hommes et des femmes issus d'une même formation. Définie ainsi, elle correspond à une ségrégation supplémentaire qui s'ajoute, en l'amplifiant ou la réduisant, à la ségrégation professionnelle d'origine éducative. Compte tenu des données utilisées, nous ne sommes pas en mesure de trancher la question des causes de cette ségrégation supplémentaire; nous ne pouvons en effet pas privilégier a priori l'explication à partir de ce qui relève des jeunes (préférences pour certaines professions, représentations positives ou négatives de certains métiers, rejets de certaines conditions de travail, autocensure dans l'accès à certaines fonctions, opportunités, contraintes familiales ou géographiques...) ou de ce qui est imputable aux entreprises (pratiques discriminatoires directes ou indirectes à l'embauche à l'égard de l'un ou l'autre sexe, volonté de «mixiser » leurs équipes, souplesse dans les conditions de travail proposées...). Cependant, l'importance et le sens de cette ségrégation apportent un éclairage complémentaire nécessaire pour cerner la part relative de la ségrégation professionnelle d'origine éducative et donc pour relativiser le poids des orientations scolaires dans les destinées professionnelles. 
Nous allons dans une première partie présenter les différents indicateurs retenus pour mesurer la ségrégation professionnelle et ses composantes, au niveau d'une profession isolée comme pour l'ensemble des emplois. La deuxième partie sera consacrée à l'estimation empirique de ces indicateurs à partir des données de l'enquête Génération 98 du Céreq. La troisième partie mettra l'accent sur l'hétérogénéité des profils ségrégatifs au sein des professions et proposera une catégorisation de ces professions selon l'intensité et l'origine de la ségrégation observée.

\section{MESURER ET DÉCOMPOSER LA SÉGRÉGATION PROFESSIONNELLE}

Cette partie présente le type de décomposition auquel nous nous sommes livrés. Elle expose les différentes grandeurs chargées de mesurer la ségrégation professionnelle et ses composantes. Elle présente également les relations que celles-ci entretiennent entre elles. Un tableau récapitulatif évitant les formalisations algébriques résume ces grandeurs en fin de section (tableau 2). Le lecteur non spécialiste pourra s'y reporter directement (page 18).

D'un point de vue algébrique, il existe plusieurs façons de mesurer la ségrégation, quelle que soit sa nature, professionnelle ou éducative (Flückiger et Silber, 1999). Inspiré de la mesure proposée par Duncan et Duncan (1955), l'indice de Karmel et Maclachlan (1988) vaut :

$$
I_{K M}=\frac{1}{2} \sum_{k=1}^{z}\left|\frac{F_{k}}{T .}-\frac{\frac{F}{T} T_{k}}{T .}\right|+\frac{1}{2} \sum_{k=1}^{z}\left|\frac{M_{k}}{T .}-\frac{\frac{M}{T} T_{k}}{T .}\right|
$$

où $\mathbf{F}_{k}, \mathbf{M}_{k}$ et $\mathbf{T}_{\mathrm{k}}$ désignent respectivement le nombre de femmes, d'hommes et le total d'individus $\left(\mathbf{T}_{\mathrm{k}}=\mathbf{F}_{k}+\mathbf{M}_{k}\right.$ ) dans la k ${ }^{\mathrm{ème}}$ catégorie (sur un total de $\mathrm{z}$ (professions ou formations)), F., M. et T. le nombre de femmes, d'hommes et le total d'individus $(\mathbf{T} .=\mathbf{F} .+\mathbf{M}$. $)$ dans la population entière. Plus frustre que d'autres indices, il a le mérite d'avoir une interprétation simple. Il récapitule l'écart existant entre la distribution des femmes et celle des hommes dans les différentes catégories et mesure donc les proportions d'hommes et de femmes qu'il faudrait déplacer pour égaliser les distributions, tout en gardant constant l'effectif total de chaque catégorie ( $\mathbf{T}_{\mathrm{k}}$ invariant). On montre (Borghans et Groot, 1999) que cet indice se ré-écrit de façon équivalente :

(2) $I_{K M}=\frac{M \cdot F}{T^{2}} \sum_{k=1}^{z}\left|\frac{F_{k}}{F}-\frac{M_{k}}{M}\right|$, en posant $\frac{M \cdot F}{T^{2}}=\mathbf{A}$

Cet indice prend des valeurs comprises entre 0 et $2 \mathbf{A}$, avec $2 \mathrm{~A} \leq 0.5$ (la valeur maximale étant atteinte si $\mathrm{F} .=$ M., i.e. on a autant de femmes que d'hommes dans la population totale étudiée).

Posons :

(3) $F_{k}^{\text {egal }}=\frac{F}{T} T_{k}$ et (4) $M_{k}^{\text {egal }}=\frac{M}{T} T_{k}=T_{k}-F_{k}^{\text {egal }}$

Ces valeurs représentent les nombres attendus d'hommes et de femmes dans la catégorie $k$ en l'absence de ségrégation. En introduisant (3) et (4) dans (1), on obtient (Borghans et Groot 1999) :

(5) $I_{K M}=\sum_{k=1}^{z}\left|\frac{F_{k}}{T}-\frac{F_{k}^{\text {egal }}}{T}\right|=\sum_{k=1}^{z}\left|S_{k}\right|$

où $\mathbf{S}_{k}$ représente la « contribution » de la catégorie $k$ à l'indice de ségrégation. Appliquée à la mesure de la ségrégation professionnelle, l'équation (5) donne l'indice suivant :

(6)

$$
\begin{aligned}
& S P=\frac{M . F_{.}}{T^{2}} \sum_{j=1}^{n}\left|\frac{F_{. j}}{F}-\frac{M . j}{M .}\right|=\sum_{j=1}^{n}\left|\frac{F_{. j}}{T}-\frac{F_{. j}^{\text {egal }}}{T .}\right|=\sum_{j=1}^{n}\left|S P_{j}\right| \\
& \text { où (7) } S P_{j}=A\left(\frac{F_{. j}}{F .}-\frac{M_{. j}}{M .}\right)=\frac{F_{. j}}{T .}-\frac{F_{. j}^{\text {egal }}}{T .}
\end{aligned}
$$

Les valeurs $\boldsymbol{S P}_{j}$ représentent les contributions des différents groupes professionnels à la ségrégation professionnelle globale. Une valeur positive de $\boldsymbol{S P}_{j}$ indique que la profession $j$ est plus féminisée que la moyenne, une valeur négative que $\mathrm{j}$ est moins féminisée que la moyenne. Ces grandeurs ne sont pas, telles quelles, directement comparables entre elles car elles intègrent déjà le poids relatif des groupes professionnels qu'elles représentent. Afin de les rendre directement comparables, les contributions des professions à la ségrégation doivent donc être pondérées par l'inverse de leur poids relatif :

(8) $S P_{j}^{r e l}=S P_{j} \frac{T}{T_{. j}}$, termes qu'on privilégiera dans la présentation des résultats. 
Appliquée cette fois-ci à la mesure de la ségrégation éducative, l'équation (5) donne alors l'indice :

(9)

$$
\begin{aligned}
& S E=\frac{M \cdot F_{.}}{T^{2}} \sum_{i=1}^{m}\left|\frac{F_{i .}}{F}-\frac{M_{i .}}{M .}\right|=\sum_{i=1}^{m}\left|\frac{F_{i .}}{T .}-\frac{F_{i .}^{\text {egal }}}{T .}\right|=\sum_{i=1}^{m}\left|S E_{i}\right| \\
& \text { où (10) } S E_{i}=A\left(\frac{F_{i .}}{F}-\frac{M_{i .}}{M .}\right)=\frac{F_{i .}}{T .}-\frac{F_{i .}^{\text {egal }}}{T .}
\end{aligned}
$$

On peut maintenant prendre en compte le fait que chacun des groupes professionnels est alimenté à sa façon par les différentes formations. On a alors :

(11) $S P_{j}=\sum_{i=1}^{m}\left(\frac{F_{i j}}{T}-\frac{F_{i j}^{e g a l}}{T}\right)$ ò̀ $F_{i j}^{\text {egal }}=\frac{F}{T} T_{i j}$ et $\boldsymbol{F}_{i j}$

représente le nombre de femmes $\left(\boldsymbol{T}_{i j}\right.$ le nombre d'individus) de la formation $i$ dans la profession $j$. On a donc :

$$
S P=\sum_{j=1}^{n}\left|\sum_{i=1}^{m}\left(\frac{F_{i j}}{T}-\frac{F_{i j}^{e g a l}}{T}\right)\right|
$$

Si on accepte l'hypothèse que l'on est face à un phénomène séquentiel où les processus d'orientation dans les formations interviennent avant les processus d'allocation dans les emplois, il devient logique de considérer que ces derniers sont en fait des processus partiellement contraints par les premiers. Réussir à exprimer ces contraintes est l'enjeu même du travail de décomposition. Il apparaît utile d'introduire une grandeur intermédiaire qui permette de faire la séparation entre les deux sources de ségrégation professionnelle. Nous reprenons ici la grandeur déjà proposée par Borghans et Groot (1999) : $F_{i j}^{\text {educ }}(i=1, \ldots m, j=1, \ldots n)$ telle que :

(13) $F_{i j}^{\text {educ }}=\frac{F_{i .}}{T_{i .}} T_{i j}$ représente pour la profession $j$ le nombre attendu de femmes issues de la formation $i$, c'est-à-dire le nombre qu'on observerait si hommes et femmes sortis d'une même formation se répartissaient dans les mêmes proportions entre les différents groupes professionnels, à effectif constant d'individus d'une formation donnée mobilisés dans une profession donnée (i.e. les $\boldsymbol{T}_{j}$ sont invariants). Nous proposons alors d'introduire (14) dans (11) et séparer ainsi un terme de ségrégation professionnelle
« héritée » de la ségrégation éducative d'un terme de ségrégation professionnelle « additionnelle », fruit de différents processus ségrégatifs agissant après la sortie de formation. Cela donne :

$$
S P_{j}=\left(\sum_{i=1}^{m}\left(\frac{F_{i j}}{T .}-\frac{F_{i j}^{\text {educ }}}{T .}\right)+\sum_{i=1}^{m}\left(\frac{F_{i j}^{\text {educ }}}{T .}-\frac{F_{i j}^{\text {egal }}}{T .}\right)\right)=S P_{j}^{M T}+S P_{j}^{\text {educ }}
$$

où :

$$
S P_{j}^{M T}=\sum_{i=1}^{m}\left(\frac{F_{i j}}{T}-\frac{F_{i j}^{e d u c}}{T .}\right) \text { est la contribution }
$$

de la profession $j$ à la SP liée au marché du travail,

(16) $S P_{j}^{\text {educ }}=\sum_{i=1}^{m}\left(\frac{F_{i j}^{\text {educ }}}{T .}-\frac{F_{i j}^{e g a l}}{T .}\right)$ est la contribution de $j$ à la SP d'origine éducative.

On a alors :

(17) $S P=\sum_{j=1}^{n}\left|S P_{j}^{M T}+S P_{j}^{\text {educ }}\right| \leq \sum_{j=1}^{n}\left|S P_{j}^{M T}\right|+\sum_{j=1}^{n}\left|S P_{j}^{\text {educ }}\right|$,

et donc :

$$
\begin{aligned}
& \text { (18) } S P \leq S P^{M T}+S P^{\text {educ }} \text { où } \\
& S P^{M T}=\sum_{j=1}^{n}\left|S P_{j}^{M T}\right| \text { et } S P^{\text {educ }}=\sum_{j=1}^{n}\left|S P_{j}^{\text {educ }}\right|
\end{aligned}
$$

Cela signifie que, calculée au niveau global, la ségrégation professionnelle finalement observée sera inférieure ou égale à l'addition des ségrégations professionnelles d'origine éducative et liée au marché du travail. En effet, chaque fois que les deux sources de ségrégation joueront en sens contraire au sein d'une profession, la composante de la ségrégation liée au marché du travail aura en fait une vertu déségrégative.

Pour un groupe professionnel donné, la relation entre les deux composantes mesurées de la ségrégation professionnelle va déterminer le genre dominant dans la profession. On peut résumer les six principaux résultats possibles concernant le processus de ségrégation professionnelle ${ }^{1}$ selon les valeurs respectives de $\mathbf{S P}, \mathbf{S P}^{M T}$ et $\mathbf{S P}^{e d u c}$ ( $c f$. tableau 1).

\footnotetext{
${ }^{1}$ En ne tenant pas compte des combinaisons incluant les égalités strictes.
} 
Tableau 1

Nature de la ségrégation professionnelle selon son origine

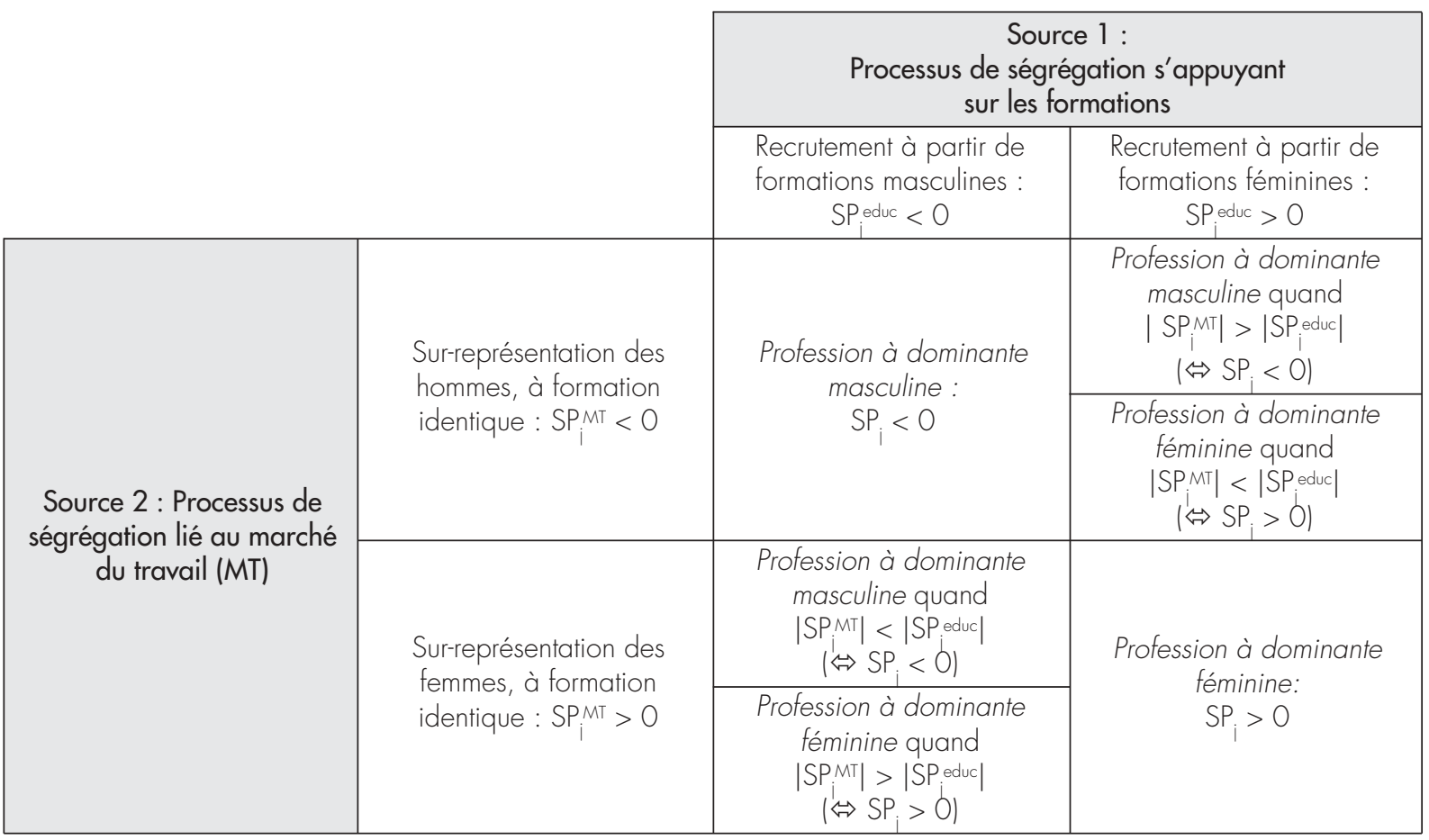

Note de lecture : $\left|\mathrm{SP}_{\mathrm{j}}^{\mathrm{MT}}\right|$ et $\left|\mathrm{SP}_{\mathrm{j}}^{\text {educ| }}\right|$ représentent les valeurs absolues de $\mathrm{SP}_{\mathrm{j}}^{\mathrm{MT}}$ et $\mathrm{SP}_{\mathrm{j}}^{\text {educ }}$.

Memento des sigles :

SP : ségrégation professionnelle calculée à partir des écarts observés dans les distributions des hommes et des femmes dans les professions. $\mathrm{SP}_{\mathrm{j}}$ : ségrégation professionnelle associée à la profession $\mathrm{j}$; il s'agit de la contribution de la profession $\mathrm{j}$ à la ségrégation professionnelle.

SPeduc : ségrégation professionnelle d'origine éducative, calculée à partir des écarts attendus dans les distributions des hommes et des femmes dans les professions, c'est-à-dire en supposant que, à formation identique, hommes et femmes se distribuent de la même façon dans les professions. $\mathrm{SP}_{\mathrm{j}}$ educ : ségrégation professionnelle d'origine éducative associée à la profession $\mathrm{j}$, soit la contribution de la profession $\mathrm{j}$ à la ségrégation professionnelle d'origine éducative.

$\mathrm{SP}^{\mathrm{мт}}$ : ségrégation professionnelle liée au marché du travail, calculée par différence entre écart observé et écart attendu dans les distributions des hommes et des femmes dans les professions.

$\mathrm{SP}_{\mathrm{j}}^{\mathrm{MT}}$ : ségrégation professionnelle liée au marché du travail associée à la profession $\mathrm{j}$, soit la contribution de la profession $\mathrm{j}$ à la ségrégation professionnelle liée au marché du travail.

Létude des relations entre ségrégation éducative (SE) et ségrégation professionnelle d'origine éducative (SPeduc) mérite une attention particulière car elle est au cour de la question cruciale de la transmission de la SE à la SP. On va voir que SPeduc peut s'interpréter comme une projection partielle ou totale de SE. En effet, on montre aisément (Couppié et Epiphane 2004b) que :

$$
\boldsymbol{O} \leq \boldsymbol{S P} \text { duc } \leq S E
$$

La condition nécessaire pour avoir l'égalité $(\mathrm{SPeduc}=\mathrm{SE})$ est que chacun des $\mathrm{n}$ groupes professionnels ne fasse appel qu'à des individus issus de formations « de même genre ». A contrario, dès lors qu'il existe au moins un groupe professionnel qui emploie des individus issus de formations « de genre opposé », on aura SPeduc < SE. De ce point de vue, le rapport $\boldsymbol{S} \boldsymbol{P}^{\text {educ }} / \boldsymbol{S E}$ va traduire la qualité de la « conversion » de la ségrégation éducative en ségrégation professionnelle au moment de l'entrée sur le marché du travail. Incidemment, ce rapport mesure la façon dont une petite variation dans la ségrégation affectant les formations se communiquera, toutes choses et comportements égaux par ailleurs, à la ségrégation affectant les professions. 
Symétriquement, par rapport à la ségrégation professionnelle prenant forme sur le marché du travail, on peut définir la ségrégation additionnelle $(\boldsymbol{S} \boldsymbol{A})$ qui récapitule, à formation identique, la totalité des différences d'allocation dans les professions entre jeunes hommes et femmes (voir Couppié et Epiphane, 2004b). Cette ségrégation additionnelle représente la ségrégation liée au marché du travail qu'on pourrait potentiellement observer pour peu que les processus de ségrégation soient systématiquement en faveur du même groupe sexué.

Le rapport $S P^{M T} / S A$ constituera alors un indicateur du degré de convergence des mécanismes ségrégatifs qui naissent sur le marché du travail ; autrement dit, il signalera dans quelle mesure, lorsqu'il y a affinité plus marquée entre un groupe professionnel et l'un des sexes, cette affinité se trouve systématiquement répétée quelle que soit la formation d'origine.

Décliné au niveau d'un groupe professionnel, on va avoir : $0 \leq \mathrm{SP}_{\mathrm{j}}^{\text {educ }} \leq \mathrm{SE}_{\mathrm{j}}$ où $S E_{j}=\sum_{i=1}^{m}\left|\frac{F_{i j}^{\text {educ }}}{T}-\frac{F_{i j}^{\text {egal }}}{T .}\right|$.
La grandeur (20) $\mathbf{S E}_{\mathrm{j}}^{\mathrm{rel}}=\mathbf{S E}_{\mathrm{j}}\left(\mathbf{T}\right.$. $\left./ \mathbf{T}_{\mathrm{rj}}\right)$ est intéressante car elle nous renseigne sur le degré de ségrégation éducative qui caractérise les formations alimentant spécifiquement le groupe professionnel $j$. Cette grandeur est directement comparable au niveau de SE qui caractérise la population entière. Le rapport $0 \leq\left(S P_{j}^{\text {educ }} / S E_{j}\right) \leq 1$ va lui nous renseigner sur la qualité de la transmission de la SE à la SP d'origine éducative dans le groupe professionnel $j$. Symétriquement, on va décliner par profession les mêmes types d'indicateurs du côté de la ségrégation professionnelle liée au marché du travail, avec (21) $\mathbf{S A}_{j}{ }_{j}^{\text {rel }}=\mathbf{S A}_{j}$ $\left(\mathbf{T} . / \mathbf{T}_{. j}\right)$ et le rapport $0 \leq\left(S P_{j}^{M T} / S A_{j}\right) \leq 1$.

Finalement, le tableau 2 résume les différentes grandeurs que nous nous proposons de calculer.

Ayant défini les différentes grandeurs qui mesurent la ségrégation professionnelle et ses composantes, nous passons maintenant à leur estimation empirique. Cette estimation s'appuie sur des données de l'enquête Génération 98 relatives à la situation de jeunes sortis du système éducatif en 1998 et qui ont été interrogés sur leur situation en avril 2001 (voir Epiphane et al. (2001) pour une présentation de l'enquête).

\section{Encadré 1 \\ Quelques remarques sur les liens entre ségrégation éducative et ségrégation professionnelle d'origine éducative à partir de cas de figures particuliers}

- Si la ségrégation éducative est absente ou quasi absente (SE proche de O), il n'y a en quelque sorte rien à " transmettre » et la ségrégation professionnelle d'origine éducative sera forcément presque nulle (SPeduc proche de O).

- Quel que soit le niveau (non nul : SE $\neq$ O) de la ségrégation éducative en amont, on ne retrouvera pas du tout de SPeduc si tous les groupes professionnels sont indifférents aux formations, c'est-à-dire que toutes les professions font appel de la même façon aux différentes formations. Statistiquement, on aurait indépendance de la distribution des formations selon les différents groupes professionnels. Cela constitue une condition suffisante mais pas nécessaire à l'absence de SPeduc. Donc, a contrario, la dépendance statistique est une condition nécessaire (mais pas suffisante) à la présence de SPeduc.

- Quel que soit le niveau (non nul : SE $\neq 0$ ) de la ségrégation éducative en amont, si chaque groupe professionnel est entièrement tributaire d'une seule formation, alors l'intégralité de la SE "passera » dans la SPeduc. (cf. par exemple le cas des professions d'infirmières, d'experts-comptables,... et plus généralement des marchés professionnels où l'accès est réglementé par la possession d'un diplôme). Cela constitue aussi une condition suffisante mais pas nécessaire à la transmission intégrale de SE en SPeduc. Donc, a contrario, pour contrecarrer le passage de la SE en SPeduc, il est nécessaire (mais pas suffisant) qu'un groupe professionnel s'appuie sur plusieurs formations différentes. 
Tableau 2

Principales grandeurs mesurant la ségrégation professionnelle et ses composantes

\begin{tabular}{|c|c|c|c|}
\hline Type de ségrégation & Sigle & Mode de calcul & Remarques \\
\hline Ségrégation professionnelle & SP & $\begin{array}{l}\text { à partir des écarts observés dans les distribu- } \\
\text { tions des hommes et des femmes dans les } \\
\text { professions }\end{array}$ & $\mathrm{SP} \leq \mathrm{SPeduc}+\mathrm{SPMT}$ \\
\hline $\begin{array}{l}\text { Ségrégation professionnelle } \\
\text { associée à la profession i }\end{array}$ & $S P_{i}$ & $\begin{array}{l}\text { contribution de la profession i à la } \\
\text { ségrégation professionnelle }\end{array}$ & $S P_{i}=S P_{i}^{\text {educ }}+S P_{i}^{M T}$ \\
\hline $\begin{array}{l}\text { Ségrégation professionnelle } \\
\text { d'origine éducative }\end{array}$ & SPeduc & $\begin{array}{l}\text { à partir des écarts attendus dans les distribu- } \\
\text { tions des hommes et des femmes dans les } \\
\text { professions, compte tenu de leurs cursus } \\
\text { scolaires différents, mais en supposant que } \\
\text { hommes et femmes issus d'une même forma- } \\
\text { tion se distribuent de la même façon dans les } \\
\text { professions }\end{array}$ & $\begin{array}{l}\text { le rapport SPeduc / SE (compris entre } \\
0 \text { et } 1 \text { ) donne la qualité de la trans- } \\
\text { formation de la ségrégation éduca- } \\
\text { tive en ségrégation professionnelle } \\
\text { d'origine éducative }\end{array}$ \\
\hline $\begin{array}{l}\text { Ségrégation professionnelle } \\
\text { d'origine éducative } \\
\text { associée à la profession i }\end{array}$ & SPeduc & $\begin{array}{l}\text { contribution de la profession i à la ségréga- } \\
\text { tion professionnelle d'origine éducative }\end{array}$ & $\begin{array}{l}\text { même interprétation que le rapport } \\
\text { précédent pour la profession j. }\end{array}$ \\
\hline $\begin{array}{l}\text { Ségrégation professionnelle } \\
\text { liée au marché du travail }\end{array}$ & SPMT & $\begin{array}{l}\text { par différence entre écarts observés et écarts } \\
\text { attendus dans les distributions des hommes et } \\
\text { des femmes dans les professions }\end{array}$ & $\begin{array}{l}\text { le rapport SPMT / SA (compris entre } \\
0 \text { et } 1 \text { ) donne le degré de conver- } \\
\text { gence des mécanismes ségrégatifs } \\
\text { qui naissent sur le marché du travail }\end{array}$ \\
\hline $\begin{array}{l}\text { Ségrégation professionnelle } \\
\text { liée au marché du travail } \\
\text { associée à la profession i }\end{array}$ & $\mathrm{SP}_{\mathrm{i}}^{\mathrm{MT}}$ & $\begin{array}{l}\text { contribution de la profession j à la ségréga- } \\
\text { tion professionnelle liée au marché du travail }\end{array}$ & $\begin{array}{l}\text { Même interprétation que le rapport } \\
\text { précédent pour la profession j. }\end{array}$ \\
\hline Ségrégation éducative & SE & $\begin{array}{l}\text { à partir des écarts observés dans les distribu- } \\
\text { tions des hommes et des femmes dans les } \\
\text { formations, au moment où ils quittaient le } \\
\text { système éducatif }\end{array}$ & $\begin{array}{l}\text { SE est la valeur maximale que peut } \\
\text { prendre SPeduc }\end{array}$ \\
\hline $\begin{array}{l}\text { Ségrégation éducative } \\
\text { associée à la profession j }\end{array}$ & $\mathrm{SE}_{\mathrm{i}}$ & $\begin{array}{l}\text { contribution de la profession j à la ségréga- } \\
\text { tion éducative }\end{array}$ & $\begin{array}{l}S_{i} \text { est la valeur maximale que peut } \\
\text { prendre SP educ }\end{array}$ \\
\hline Ségrégation additionnelle & SA & $\begin{array}{l}\text { par différence entre écarts observés et écarts } \\
\text { attendus dans les distributions des hommes et } \\
\text { des femmes dans les professions, à l'issue de } \\
\text { chacune des formations }\end{array}$ & $\begin{array}{l}\text { SA est la valeur maximale que peut } \\
\text { prendre SPMT }\end{array}$ \\
\hline $\begin{array}{l}\text { Ségrégation professionnelle } \\
\text { associée à la profession i }\end{array}$ & $S A_{i}$ & $\begin{array}{l}\text { contribution de la profession i à la ségréga- } \\
\text { tion additionnelle }\end{array}$ & $\begin{array}{l}\text { SA est la valeur maximale que peut } \\
\text { prendre SPMT }\end{array}$ \\
\hline
\end{tabular}

\section{LES COMPOSANTES DE LA SÉGRÉGATION PROFESSIONNELLE : RÉSULTATS EMPIRIQUES}

Nous présentons d'abord les résultats empiriques obtenus au niveau de l'ensemble de la population étudiée dans le calcul de la ségrégation professionnelle et de ses composantes. Nous détaillons ensuite la variété des configurations observées au niveau des groupes professionnels.

\section{Les composés de la ségrégation professionnelle au niveau global}

Appliqué aux données de l'enquête Génération 98 du Céreq, un découpage en 79 groupes professionnels ${ }^{2}$ et

277 groupes professionnels stricto sensu et 2 groupes représentant d'une part les chômeurs et d'autre part tous les inactifs. Pour une présentation des 74 types de formation, voir Couppié et Epiphane (2004a). 
74 types de formation donne, sur la situation à la date de l'enquête, un indice de ségrégation professionnelle de $22,7 \%$. En d'autres termes, pour obtenir une répartition uniforme des hommes et des femmes dans l'ensemble des groupes professionnels, il faudrait réaffecter presque le quart d'entre eux. La ségrégation au sein de la génération 98 se situe à mi-chemin entre les deux scénarii extrêmes : une mixité parfaite (où l'indice de ségrégation serait égal à 0 ) et une ségrégation complète (où cet indice serait égal à $50 \%)$. De plus :

- Selon le schéma de décomposition algébrique de la ségrégation professionnelle présenté plus haut, cette ségrégation est imputable, pour pratiquement les deux tiers, aux effets de la ségrégation éducative et pour un gros tiers à la ségrégation supplémentaire prenant corps sur le marché du travail. Il apparaît donc que notre travail de décomposition n'est pas vain.

- Comme on pouvait s'y attendre, les disparités d'orientation entre les filles et les garçons dans la formation initiale - qui étaient importantes au sein de la génération $98(\mathrm{SE}=25,6 \%)$ - ont un impact conséquent sur la répartition différenciée des unes et des autres dans les professions $\left(\mathrm{SP}^{\mathrm{educ}}=14,7 \%\right)$. Cependant, l'état des dépendances entre formations et groupes professionnels est tel que la « conversion » de la ségrégation éducative en ségrégation professionnelle apparaît loin d'être complète (SPeduc / SE = 57\%).

- La ségrégation supplémentaire à l'œuvre sur le marché du travail, si elle pèse quantitativement moins, est néanmoins conséquente ( $\mathrm{SP}^{\mathrm{MT}}=9,1 \%$ ). De plus, les mécanismes ${ }^{3}$ sexuellement différenciés d'appariement entre individus et emplois indépendants des qualifications scolaires acquises que traduit ce type de ségrégation apparaissent relativement cohérents par profession, puisqu'ils tendent à privilégier assez systématiquement l'un ou l'autre sexe $\left(S P^{M T} / S A=70 \%\right)$.

- Des mécanismes de compensation entre les deux sources de ségrégation existent $(\mathrm{SP}$ educ $+\mathrm{SP} \mathrm{MT}>\mathrm{SP})$

\footnotetext{
${ }^{3}$ Sans que l'on suppose a priori ce qui relève des jeunes (préférences pour certaines professions, représentations positives ou négatives de certains métiers, rejets de certaines conditions de travail, autocensure dans l'accès à certaines fonctions, opportunités, contraintes familiales ou géographiques...), de ce qui est imputable aux entreprises (discrimination à l'égard de l'un ou l'autre sexe, volonté de «mixiser» leurs équipes, souplesse dans les conditions de travail proposées...)
}

mais apparaissent globalement faibles ((SPeduc + $\left.\mathrm{SP}^{\mathrm{MT}}\right) / \mathrm{SP}=1,05$ ).

Rappelons que l'estimation de la ségrégation professionnelle et de ses composantes dépend des choix de nomenclature faits ; plus les nomenclatures d'emploi et de formation seront fines, plus élevée pourra être la ségrégation professionnelle (voir encadré 2). La mesure de la ségrégation professionnelle reste aussi contingente au moment où elle est mesurée - ici, trois ans après la sortie de formation. L'encadré 2 éclaire aussi cet aspect des choses.

Après ce bref aperçu des résultats d'ensemble, nous pouvons maintenant caractériser plus en détail l'hétérogénéité des phénomènes de ségrégation entre groupes professionnels.

\section{Souvent, la ségrégation liée au marché du travail renforce la ségrégation $d^{\prime}$ 'origine éducative}

Une majorité de groupes professionnels (43 contre 34) «privilégie » dans ses recrutements, les jeunes issus des formations féminines, une plus grande majorité encore (48 contre 29) «favorise », à formation identique, les jeunes hommes par rapport aux jeunes femmes. Au final, parmi les 77 groupes professionnels, 33 accueillent plus de femmes que la moyenne et 44 plus d'hommes que la moyenne.

Le cas de figure où les deux sources de ségrégation se cumulent est le plus fréquent. $38 \%$ des groupes professionnels (29 sur 77) représentent ainsi des professions à dominante masculine qui recrutent majoritairement des jeunes issus de formations masculines et, à formation identique, privilégient les garçons par rapport aux filles (ouvriers du bâtiment, ingénieurs en informatique, policiers...). De façon symétrique, $31 \%$ des groupes professionnels (24 sur 77) représentent les professions à dominante féminine qui recrutent majoritairement des jeunes issus de formations féminines et, à formation identique, privilégient les filles par rapport aux garçons (employées de maison, aidessoignantes, secrétaires...). Ces deux cas de figures rassemblent donc environ $70 \%$ de l'ensemble des groupes professionnels et $78 \%$ des emplois. Dans les deux cas, la ségrégation est alimentée à la fois par la ségrégation d'origine éducative et par la ségrégation liée au marché du travail, chacune des deux étapes contribuant de façon variable au phénomène. 


\section{Encadré 2 \\ Impacts du choix de la nomenclature de professions retenue et du moment choisi de l'observation sur la mesure de la ségrégation professionnelle}

Notre travail se base sur une nomenclature de groupes professionnels en 77 postes (plus un poste pour les chômeurs et un poste pour les inactifs). Logiquement, plus on travaille sur une nomenclature détaillée, plus on saisit de différences entre hommes et femmes et donc plus on saisit de ségrégation professionnelle. Quel degré d'affinement faut-il retenir pour saisir l'essentiel du phénomène? Les découpages les plus simples (comme la position sociale en 6 postes) sont à rejeter car ils amputent trop la mesure de la ségrégation professionnelle par rapport au découpage le plus complet. En revanche, les différences n'apparaissent pas à première vue notables quand on considère la catégorie socioprofessionnelle ICS de l'Insee en 31 postes), notre nomenclature de référence (77 postes) ou la nomenclature des Professions et Catégories Socioprofessionnelles détaillée (PCS de I'Insee en 433 postes) (Couppié et Epiphane 2004b). Cependant, une estimation de l'écart-type de nos mesures de SP par une procédure de bootstrap indique que la mesure donnée à partir de la CS est inférieure aux deux autres, notamment parce qu'elle réduit la ségrégation professionnelle prenant forme sur le marché du travail, objet central de notre analyse. L'écart des mesures entre notre nomenclature et la PCS détaillée se situe à la limite des seuils de significativité.

Concernant le degré de stabilité de nos mesures de ségrégation professionnelle au fil du parcours d'insertion, on observe globalement une augmentation de la ségrégation professionnelle entre avril 1999 (10 mois après la sortie du système éducatif) et avril 2001 (34 mois après la sortie), et particulièrement de la ségrégation professionnelle d'origine éducative. Quant à la ségrégation professionnelle liée au marché du travail, elle suit l'évolution inverse et apparaît être plus forte en début de parcours qu'en fin de parcours (Couppié et Epiphane, 2004b). II existe différentes façons d'interpréter ce résultat. Par exemple, selon le modèle de la file d'attente, les jeunes qui n'accèderaient pas rapidement aux postes pour lesquels ils se sont préparés à l'école accepteraient en attendant - et en accumulant de l'expérience professionnelle - des emplois transitoires, souvent moins qualifiés et/ou hors de leur domaine de qualifications. Ces emplois transitoires incorporeraient alors plus facilement de la ségrégation professionnelle liée au marché du travail en générant une polarisation conforme aux stéréotypes de sexes et moins dépendante des cursus scolaires.

Les mesures des différentes formes de ségrégation professionnelle calculées sur l'ensemble des parcours se révèlent très nettement inférieures à ce qu'on a mesuré à chacune des deux extrémités des parcours observés. En effet, quelques groupes professionnels ne gardent pas un genre constant sur les trois premières années de vie active. Pour ces groupes, l'opération visant à agréger dans un même ensemble les séquences situées en début de parcours - sur-représentant un des deux sexes - avec des séquences plus tardives - sur-représentant l'autre sexe - revient à gommer une partie de la ségrégation professionnelle observable à un moment donné. Ainsi, en comparant les situations en avril 1999 et avril 200 1, 7 groupes professionnels ont changé de genre. Ces changements concernent des catégories généralement assez peu ségréguées aux deux dates. Cependant, le groupe qui joue un rôle prépondérant dans la baisse des mesures de ségrégation est le groupe des inactifs, catégorie disparate qui accueille autant les jeunes en reprise d'études ou en formation que les jeunes effectuant leur service national - essentiellement des hommes, très représentés en avril 1999 - ou que les autres inactifs. 
A contrario, dans les 24 autres groupes professionnels, les effets respectifs des ségrégations d'origine éducative et liée au marché du travail s'opposent. Dans la moitié des cas, les effets de la ségrégation d'origine éducative sont les plus forts, dans l'autre moitié, ceux de la ségrégation liée au marché du travail l'emportent. Par ailleurs, quel que soit le taux de féminisation du groupe professionnel, dans plus de trois quarts de ces groupes (soit 19 sur 24), la ségrégation d'origine éducative favorise les jeunes issus des spécialités féminines alors que la ségrégation liée au marché du travail favorise les jeunes hommes. C'est donc dans seulement un quart des cas (5 sur 24) que la ségrégation d'origine éducative favorise les jeunes issus des spécialités masculines et que la ségrégation liée au marché du travail favorise les jeunes femmes.

Ainsi, pour la moitié de ces 24 groupes professionnels, la ségrégation d'origine éducative domine malgré un recrutement qui privilégie le sexe minoritaire dans les formations où elles puisent. Par exemple, le groupe professionnel des «professeurs certifiés ou agrégés », plutôt féminin $(61 \%)$, recrute les jeunes majoritairement issus de formations féminines tout en privilégiant, à formation identique, les jeunes hommes. Le cas de figures où le recrutement privilégie les jeunes hommes issus de spécialités féminines est plus fréquent que celui où les jeunes femmes dotées d'une formation masculine sont sur sélectionnées. Il est à noter que la correction apportée par la ségrégation liée au marché du travail est généralement faible.

En revanche, pour l'autre moitié de ces groupes professionnels, la ségrégation prenant forme sur le marché du travail compense et dépasse les effets de la ségrégation d'origine éducative. Par exemple, le groupe des «cadres de la banque et des assurances" mobilise un peu plus de jeunes issus de formations féminines, mais surreprésente largement - à formation égale - les hommes dans les recrutements. Dans ces cas-là, en règle générale, la ségrégation d'origine éducative y est très faible, voire inexistante. Enfin, dans la quasi-totalité des cas (11 sur 12), il s'agit de groupes professionnels qui privilégient, via la ségrégation liée au marché du travail, les hommes. Le seul et unique groupe professionnel où les femmes sont surreprésentées, alors même que le vivier est plutôt constitué de jeunes issus de formations masculines, est le groupe des nettoyeurs, c'est-à-dire les ouvriers travaillant dans le secteur de la propreté.

\section{GENRE ET ENGENDREMENT : UNE NOUVELLE TYPOLOGIE DES PROFESSIONS}

Notre but est de pointer l'importance respective des deux étapes de formation de la ségrégation professionnelle et de souligner l'hétérogénéité des processus ségrégatifs. À cet effet, nous pouvons classer les différentes professions en prenant en compte non seulement l'intensité de la ségrégation observée, mais aussi le poids des différentes sources de ségrégation. On définit ainsi une nomenclature en huit catégories théoriquement possibles. En premier lieu, pour les professions fortement ségréguées (donc « féminines » ou « masculines »), on oppose à partir du rapport SPeduc / SPMT les catégories dont la ségrégation est principalement d'origine éducative ${ }^{4}$, principalement liée au marché du travail ${ }^{5}$ ou est alimentée de façon cumulative par les deux sources. En second lieu, pour les professions «faiblement» ségréguées (donc « mixtes »), on distingue celles qui sont mixtes du fait d'une absence relative de ségrégation dans les deux étapes de celles pour lesquelles la mixité est le fruit de processus ségrégatifs contraires qui tendent à se compenser ${ }^{6}$. Les tableaux suivants présentent les nouvelles catégories obtenues. Les groupes professionnels dont la ségrégation est principalement liée au marché du travail constituent un ensemble peu fourni, exclusivement composé de groupes professionnels masculins. Ainsi, le type des « groupes professionnels féminins dont la ségrégation prend principalement forme sur le marché du travail » n'apparaît pas, compte tenu des seuils fixés.

\footnotetext{
${ }^{4} \mathrm{Si}$ la ségrégation d'origine éducative représente au moins le double (en valeur absolue) de la ségrégation liée au marché du travail.

${ }^{5}$ Inversement, si la ségrégation liée au marché du travail représente au moins le double (en valeur absolue) de la ségrégation d'origine éducative.

${ }^{6}$ Les résultats de ces découpages sont bien sûr contingents à la façon dont on délimite les catégories et reposent en partie sur les valeurs des seuils ad hoc choisis. Cependant, on a cherché à limiter au maximum les effets de seuil. Ainsi, pour définir les groupes professionnels «mixtes », c'est-à-dire «faiblement» ségrégués, on a complété un critère de choix raisonné avec un critère de coupure «naturelle » présente dans les données; le niveau retenu de ségrégation professionnelle ne doit pas dépasser $13 \%$, la part des femmes étant comprise entre $36 \%$ et $62 \%$.
} 
Le tableau 3 présente quelques résultats d'ensemble de cette typologie. Il met en évidence qu'en moyenne trois groupes professionnels sur dix sont, au sens de notre classement, mixtes (un petit quart des emplois), quatre groupes professionnels sur dix sont « masculins » (40\% des emplois) et trois sur dix « féminins » (pour presque autant d'emplois). Le genre des groupes professionnels apparaît ne pas être socialement neutre. Ainsi, les deux types mixtes sont en moyenne d'un niveau social beaucoup plus élevé que les types plus ségrégués. Les catégories sociales des cadres et les professions intermédiaires y représentent ensemble pratiquement les trois-quarts des emplois de ces types mixtes. À l'inverse, ces deux catégories sociales réunies plafonnent en général au tiers des emplois des différents types masculins ou féminins (à l'exception notable du type des professions féminines dont la ségrégation y est principalement d'origine scolaire, dont $62 \%$ des emplois sont classés professions intermédiaires).

Les professions à dominante féminine sont, deux fois sur trois, marquées par une ségrégation construite en deux temps, alors que la ségrégation au sein des professions à dominante masculine est dans la majorité des cas principalement d'origine éducative. Cette observation peut être mise en parallèle avec le constat maintes fois évoqué de relations entre les formations et les emplois plus étroites dans les professions industrielles (très souvent masculines) que dans les profes- sions tertiaires (assez fréquemment féminines ou mixtes). L'exemple du recrutement des secrétaires permet d'illustrer le mécanisme à l'œuvre. Cette catégorie s'alimente largement au-delà des formations de secrétariat - extrêmement féminisées - et va intégrer des jeunes femmes issues de formations tertiaires ou générales, féminisées mais de façon moins clivée (Couppié et Lopez, 2003).

De façon plus détaillée, on peut caractériser chacun des sept types présents (voir tableau 4) :

- La catégorie A réunit des professions masculines pour lesquelles la ségrégation est principalement d'origine éducative. Ces professions se caractérisent par une importante ségrégation professionnelle d'origine éducative, d'autant plus forte que non seulement les formations qui alimentent les professions de ce type sont elles-mêmes fortement sexuées, mais aussi et que cette forte ségrégation éducative se convertit en majeure partie en ségrégation professionnelle d'origine éducative. La ségrégation supplémentaire, qui joue ici souvent un rôle résiduel, accentue la première. Ces professions ont en commun d'être directement en lien avec la production de biens dans l'industrie. Ainsi, à côté des nombreux groupes professionnels ouvriers de type industriel ou artisanal (qualifiés ou non), la catégorie accueille les groupes de techniciens de l'industrie, des contremaîtres et agents de maîtrise et des ingénieurs (hors ingénieurs informatique).

Tableau 3

Poids et composition des types professionnels par sexe et position professionnelle

\begin{tabular}{|c|c|c|c|c|c|c|c|}
\hline \multirow[t]{2}{*}{ Type professionnel } & \multirow{2}{*}{$\begin{array}{c}\text { Nombre de } \\
\text { groupes } \\
\text { professionnels }\end{array}$} & \multirow{2}{*}{\begin{tabular}{|c|} 
Effectifs \\
$(\%)$
\end{tabular}} & \multicolumn{4}{|c|}{ Répartition par position professionnelle $(\%)$} & \multirow{2}{*}{$\begin{array}{c}\text { Taux de } \\
\text { féminisation } \\
(\%)\end{array}$} \\
\hline & & & Cadres & \begin{tabular}{|c|} 
Professions \\
intermédiaires
\end{tabular} & Autres & Total & \\
\hline "Masculin d'origine éducative " $[\mathrm{A}]$ & 18 & 23 & 8 & 24 & 68 & 100 & 14 \\
\hline «Masculin d'origine double»[B] & 8 & 13 & 18 & 12 & 69 & 100 & 18 \\
\hline "Masculin lié au marché du travail » [C] & 5 & 4 & 17 & 18 & 65 & 100 & 21 \\
\hline «Féminin d'origine éducative » [D] & 8 & 12 & 5 & 62 & 34 & 100 & 79 \\
\hline «Féminin d'origine double » $[E]$ & 15 & 25 & 0 & 21 & 79 & 100 & 78 \\
\hline "Mixte par absence de ségrégation » [G] & 13 & 15 & 31 & 39 & 30 & 100 & 49 \\
\hline «Mixte par compensation » $[\mathrm{H}]$ & 10 & 8 & 45 & 33 & 21 & 100 & 49 \\
\hline Ensemble & 77 & 100 & 14 & 29 & 57 & 100 & 46 \\
\hline
\end{tabular}

Note de lecture : le type professionnel «masculin d'origine éducative » ([A]) regroupe 18 groupes professionnels qui représentent $23 \%$ des emplois occupés en avril 2001. Toujours dans ce type professionnel, $8 \%$ des emplois sont des emplois de cadres et $14 \%$ des emplois sont occupés par des femmes.

Source : Céreq, génération 98 - interrogation 2001 
- La catégorie B regroupe les professions masculines pour lesquelles la ségrégation est à la fois d'origine éducative et liée au marché du travail. Par rapport à la catégorie précédente, la ségrégation professionnelle d'origine éducative y est légèrement moins forte. En revanche, la ségrégation professionnelle prenant corps sur le marché du travail y est plus importante en raison d'une ségrégation supplémentaire supérieure qui joue, à diplôme égal, systématiquement en faveur des garçons. Ces professions apparaissent moins directement liées à la production physique de biens dans l'industrie et concernent souvent des activités connexes comme la logistique ou le transport.

- La catégorie $\mathrm{C}$ agrège des professions masculines pour lesquelles la ségrégation prend essentiellement forme sur le marché du travail. En effet, ces professions apparaissent ouvertes presque autant aux formations féminines qu'aux formations masculines, annulant les mécanismes ségrégatifs scolaires. Pour autant, d'importants processus ségrégatifs liés au marché du travail conduisent à surreprésenter, à formation identique, fortement les hommes. Peu nombreux, ces groupes professionnels correspondent à des activités qui ne renvoient pas forcément à un cursus spécifique de la formation initiale ou pour lesquels des voies alternatives existent et sont développées. L'exemple typique de ces groupes professionnels est le groupe des employés de la police, de la gendarmerie et des agents de sécurité.

- La catégorie D isole, au sein des professions féminines, un petit noyau caractérisé par une ségrégation professionnelle principalement d'origine éducative. L'importance de la ségrégation professionnelle d'origine éducative est ici accompagnée d'une ségrégation professionnelle liée au marché du travail faible. Elle est composée pour l'essentiel des professions tertiaires « réglementées », dont l'accès est conditionné à la possession d'un diplôme ${ }^{7}-$ à l'exemple des métiers de la coiffure et de l'esthétique ou de nombreux groupes professionnels de la santé et du social (aides-soignantes, infirmières, cadres du para-médical, assistantes sociales,...) ou spécifiques à un secteur «à statut», où les

\footnotetext{
7 Voir Couppié et Lopez (2003) pour un commentaire des spécificités de ce type de professions au niveau de la catégorie sociale des employés.
}

pratiques de recrutement et de gestion de la maind'œuvre sont fortement codifiées et encadrées par les conventions collectives ${ }^{8}$, en l'occurrence ici le secteur de la banque et des assurances.

- La catégorie E rassemble la majorité des professions féminines, caractérisées par une ségrégation professionnelle construite en deux étapes. S'appuyant sur une base relativement large de formations, le plus souvent féminines, elles surreprésentent assez systématiquement les filles, à formation égale. On y retrouve la majeure partie des groupes professionnels classés employés, du privé comme du public. S'y joignent quelques groupes professionnels classés professions intermédiaires mais aucun groupe classé cadre.

- La catégorie $\mathrm{G}^{9}$ réunit les professions plutôt mixtes, caractérisées par une relative absence de ségrégation dans les deux phases. Mais ce résultat est davantage le fruit d'une mauvaise transmission de la ségrégation éducative que d'une alimentation via des formations réellement mixtes. De même, c'est moins l'absence de ségrégation supplémentaire qu'une absence de processus unilatéralement favorable à un des deux sexes qui explique un niveau faible de ségrégation professionnelle liée au marché du travail. Ainsi, les jeunes recrutés sur des emplois de cadres de gestion viennent, à parts à peu près égales, de formations "féminines » et de formations «masculines». De plus, quand on passe en revue les différentes formations alimentant ce groupe, tantôt les hommes et tantôt les femmes y sont surreprésentés.

- Enfin, la catégorie H regroupe les professions plutôt mixtes, pour lesquelles une des deux sources de ségrégation contrebalance l'autre et l'empêche de polariser la profession vers un des deux genres. À une exception près, on observe une ségrégation professionnelle d'origine éducative favorable aux femmes, compensée par une ségrégation professionnelle liée au marché du travail favorable aux hommes. Le niveau de compensation est variable : partiel (professeurs certifiés), total (professions de l'information, des arts et du spectacle), ou même supérieur (ONQ - ouvriers non qualifiés - divers).

\footnotetext{
${ }^{8}$ Voir Clémenceau et Géhin (1983) pour une définition des typologies de secteurs.

${ }^{9}$ La catégorie $\mathrm{F}$, théoriquement possible, des professions féminines pour lesquelles la ségrégation est principalement construite sur le marché du travail est, dans la pratique, vide.
} 
Tableau 4

Illustration des types différents de professions au regard de la ségrégation professionnelle

\begin{tabular}{|c|c|c|c|c|c|c|c|}
\hline \multirow[t]{3}{*}{ Groupes professionnels } & \multirow[t]{3}{*}{$\begin{array}{l}\text { Taux de } \\
\text { féminisation }\end{array}$} & \begin{tabular}{|c|} 
Ségrégation \\
professionnelle \\
observée
\end{tabular} & \begin{tabular}{|c|} 
Ségrégation \\
professionnelle \\
d'origine \\
éducative
\end{tabular} & \begin{tabular}{|c|} 
Ségrégation \\
professionnelle \\
liée au marché \\
du travail
\end{tabular} & $\begin{array}{l}\text { Ségrégation } \\
\text { éducative }\end{array}$ & $\begin{array}{l}\text { Ségrégation } \\
\text { additionnelle }\end{array}$ & \multirow[t]{2}{*}{$\begin{array}{c}\text { Rapport } \\
\mid S^{\prime} \text { educ } \\
{ }^{\prime} \mathrm{SE}_{i}\end{array}$} \\
\hline & & $\mathrm{SP}_{\mathrm{i}}$ & SPeduc & SPMT & $\mathrm{SE}_{\mathrm{i}}$ & $\mathrm{SA}_{\mathrm{i}}$ & \\
\hline & & (1) & (2) & (3) & $(4)$ & $\mid(5)$ & $(2) /(4)$ \\
\hline \multicolumn{8}{|c|}{ Masculin avec ségrégation principalement d'origine éducative $[\mathrm{A}]$} \\
\hline OQ Métallurgie, Mécanique & 7,5 & \begin{tabular}{|l|l|}
$-41,5$ \\
\end{tabular} & $-36,0$ & $-5,5$ & 40,3 & 6,9 & 89,1 \\
\hline Techniciens BTP & 20,5 & $-28,5$ & $-27,6$ & $-0,9$ & 32,1 & 14,0 & 86,1 \\
\hline $\begin{array}{l}\text { Autres Ingénieurs } \\
\text { (sauf informatique, chimie) }\end{array}$ & 20,8 & $-28,2$ & $-19,5$ & $-8,7$ & 23,8 & 10,2 & 81,7 \\
\hline \multicolumn{8}{|c|}{ Masculin avec ségrégations d'origine éducative et liée au marché du travail [B] } \\
\hline Chauffeurs et $O Q$ transport & 7,3 & \begin{tabular}{|l|l|}
$-41,7$ \\
\end{tabular} & \begin{tabular}{|l|}
$-18,8$ \\
\end{tabular} & $-22,8$ & 28,9 & 22,9 & 65,3 \\
\hline Magasiniers & 19,2 & $-29,8$ & $-10,9$ & $-18,9$ & 27,3 & 21,9 & 40,1 \\
\hline Ingénieurs informatique & 21,0 & $-28,0$ & $-17,8$ & $-10,1$ & 21,9 & 11,1 & 81,2 \\
\hline \multicolumn{8}{|c|}{ Masculin avec ségrégation principalement liée au marché du travail [C] } \\
\hline $\begin{array}{l}\text { Police, Armée, Gendarmerie } \\
\text { (Cadres et PI) }\end{array}$ & 10,4 & $-38,6$ & $-9,3$ & $-29,3$ & 23,2 & 35,6 & 40,1 \\
\hline Technico-commerciaux & 20,5 & $-28,5$ & $-6,5$ & $-22,0$ & 18,8 & 25,9 & 34,5 \\
\hline $\begin{array}{l}\text { Police, Gendarmerie, } \\
\text { Agents de sécurité (Employés) }\end{array}$ & 20,6 & $-28,4$ & $-6,1$ & $-22,3$ & 24,7 & 23,5 & 24,7 \\
\hline \multicolumn{8}{|c|}{ Mixte où les deux origines de la ségrégation sont faibles [G] } \\
\hline Ingénieurs de la chimie & 44,4 & \begin{tabular}{|l|}
$-4,6$ \\
\end{tabular} & $-4,0$ & $-0,6$ & 12,3 & 16,8 & 32,8 \\
\hline Cadres de Gestion & 49,2 & 0,2 & 0,8 & $-0,5$ & 12,2 & 10,3 & 6,3 \\
\hline Nettoyeurs & 52,8 & 3,8 & $-1,0$ & 4,8 & 28,1 & 10,5 & 3,7 \\
\hline \multicolumn{8}{|c|}{ Mixte où les deux origines de la ségrégation se compensent $[\mathrm{H}]$} \\
\hline Cadres Banque, Assurances & 38,6 & \begin{tabular}{|l|}
$-10,4$ \\
\end{tabular} & 3,4 & $-13,9$ & 8,6 & 19,4 & 40,2 \\
\hline Employés PTT & 46,6 & $-2,4$ & 6,1 & $-8,5$ & 20,6 & 19,3 & 29,4 \\
\hline Prof. certifiés et agrégés & 61,4 & 12,4 & 15,2 & $-2,8$ & 19,1 & 8,2 & 79,4 \\
\hline \multicolumn{8}{|c|}{ Féminin avec ségrégation principalement d'origine éducative [D] } \\
\hline Coiffeurs, Esthéticiens & 91,7 & \begin{tabular}{|l|}
42,7 \\
\end{tabular} & 36,0 & 6,7 & 37,1 & 7,4 & 97,2 \\
\hline $\operatorname{lnfirmiers}\left({ }^{*}\right)$ & 83,5 & 34,5 & 32,6 & 1,8 & 32,8 & 2,2 & 99,5 \\
\hline Employés Banque, Assurances & 71,4 & 22,4 & 15,9 & 6,5 & 18,5 & 11,8 & 85,8 \\
\hline \multicolumn{8}{|c|}{ Féminin avec ségrégations d'origine éducative et liée au marché du travail $[\mathrm{E}]$} \\
\hline Employés Compta-Finance & 76,7 & 27,7 & 17,0 & 10,8 & 18,9 & 12,0 & 89,5 \\
\hline $\begin{array}{l}\text { Employés de bureau de la } \\
\text { Fonction publique }\end{array}$ & 73,1 & 24,1 & 14,6 & 9,5 & 22,5 & 12,9 & 65,0 \\
\hline PI Gestion-Comptabilité & 72,4 & 23,4 & 10,7 & 12,7 & 18,1 & 17,5 & 58,8 \\
\hline
\end{tabular}

Note de lecture : (*) Pour les professions féminines avec ségrégation principalement d'origine éducative (D), le groupe professionnel des infirmiers connaît un niveau de ségrégation professionnelle supérieur à la moyenne (34,5\%, une valeur négative indiquerait une ségrégation professionnelle à l'avantage des hommes) puisqu'il est composé à 83,5\% de femmes. Les formations qui l'alimentent sont féminisées puisque la ségrégation professionnelle d'origine éducative y atteint $32,6 \%$. À l'inverse, les phénomènes de surreprésentation des femmes par rapport aux hommes, à formation identique, sont très limités puisque la ségrégation professionnelle liée au marché du travail y est très faible $\left(\mathrm{SP}^{\mathrm{MT}}=1,8 \%\right)$. La conversion de la ségrégation éducative $\left(\mathrm{SE}_{\mathrm{j}}=32,8 \%\right)$ en ségrégation professionnelle d'origine éducative y est remarquable puisque le rapport $\mathrm{SP}_{\mathrm{j}} \mathrm{educ} / \mathrm{SE}_{\mathrm{j}}$ vaut $99,5 \%$, très proche du maximum de $100 \%$.

Source : Céreq, génération 98 - interrogation 2001. 
Le tableau 4 donne quelques exemples des résultats de décomposition de la ségrégation professionnelle pour des professions illustratives des différents types (pour des résultats plus complets, voir Couppié et Epiphane, 2004a et b).

La question de la robustesse d'une telle typologie se pose. Peu d'évolutions sont significatives quand on mesure la ségrégation à différentes dates. On constate que 10 groupes professionnels sur 77 glissent dans une catégorie adjacente quand on compare la ségrégation professionnelle mesurée en avril 1999 à celle mesurée en avril 2001 (Couppié et Epiphane, 2004b). Il s'avère donc que, mis à part quelques effets de seuils inhérents à toute catégorisation, cette typologie est consolidée.

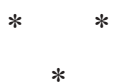

À l'instar des travaux sur le poids de l'origine sociale dans les destinées professionnelles, la décomposition de la ségrégation professionnelle entre hommes et femmes proposée ici démontre combien les modes d'allocation des individus dans les différents groupes professionnels ne prolongent pas mécaniquement les résultats de l'allocation des individus dans les formations induits par les orientations sexuellement clivées. En permettant de mesurer la part relative de la ségrégation professionnelle imputable à ces orientations scolaires, cette décomposition souligne le rôle important d'un processus ségrégatif supplémentaire indépendant des cursus scolaires. Une analyse de la ségrégation professionnelle des hommes et des femmes sur le marché du travail reposant sur la seule explication d'une simple reproduction dans le système productif de la ségrégation opérée en amont sur les bancs de l'école est donc incomplète. De plus, la décomposition opérée souligne l'hétérogénéité, entre groupes professionnels, du poids relatif de chacune des deux sources de la ségrégation professionnelle. Il semble donc plus heuristique de considérer la ségrégation professionnelle comme un processus en deux étapes.

Plus précisément, l'approche que nous avons proposée permet, d'une part, de développer des outils d'évaluation spécifiques à chacune de ces étapes pour des groupes professionnels donnés. Ainsi, le poids de la ségrégation professionnelle d'origine éducative tout comme le rapport qu'elle forme avec la ségrégation éducative apparaissent être des indicateurs utiles pour anticiper le degré de « réponse » d'une profession à des mesures visant à favoriser l'orientation scolaire d'un sexe dans des formations qui alimentent cette même profession. De ce point de vue, des groupes professionnels tels que les infirmières, les ingénieurs ou les techniciens du BTP - qui ont en commun une valeur élevée de SPeduc, un rapport SPeduc / SE proche de 1 et un rapport SPeduc / SPMT nettement supérieur 1 - semblent a priori des cibles réceptives à de telles politiques éducatives. À l'inverse, des groupes professionnels tels que celui des chauffeurs et autres ouvriers qualifiés des transports, ou celui des agents des métiers de la sécurité (police, armée, sécurité privé) - qui ont en commun une valeur faible de $\mathrm{SP}$ educ, un rapport SPeduc/SE nettement inférieur à $50 \%$ et un rapport SPeduc / SPMT largement inférieur à 1 - ne seraient que peu affectés par ces politiques, en l'état actuel de la carte des formations qui les alimentent.

D'autre part, ce type d'approche permet de souligner l'intérêt de politiques publiques en faveur de l'égalité professionnelle des hommes et des femmes plus actives sur le versant du marché du travail. La mise en évidence, pour certains groupes professionnels tels que les chauffeurs et ouvriers du transport ou les métiers de la sécurité, d'une composante forte de ségrégation professionnelle liée au marché du travail - signe d'une convergence de processus ségrégatifs à l'œuvre dans la phase d'allocation des individus dans les emplois - devrait conduire à s'interroger sur les raisons de cette polarisation d'une profession autour d'un sexe : poids des représentations sociales et sexués du métier, pratiques discriminantes des employeurs, barrières à l'embauche, influence des conditions de travail proposées, incidence des relations professionnelles qui se nouent au sein des collectifs de travail, etc.

La diversification des orientations féminines ne peut, à elle seule, résoudre le problème de l'inégale répartition des hommes et des femmes dans les professions. Par ailleurs, s'il faut sans aucun doute poursuivre les efforts en ce domaine, il s'agit aussi d'interroger les politiques de recrutement des employeurs et, plus globalement, les processus de distribution des individus - hommes et femmes - dans les différentes professions. Si certaines professions résistent plus que d'autres à la mixité, c'est non seulement du côté du système éducatif mais aussi, pour une part non négligeable, du côté du marché du travail qu'il faut regarder. 
Baudelot C., Establet R. (1992), Allez les filles, Éditions du Seuil.

Borghans L., Groot L. (1999), "Educational presorting and occupational segregation", Labour Economics, $\mathrm{n}^{\circ}$ 6, pp. 375-395.

Clémenceau P., Géhin J.-P. (1983), « Le renouvellement de la main-d'œuvre dans les secteurs : quelles conséquences pour l'accès des jeunes aux emplois? ॥ Formation Emploi, avril-juin, n 2, pp. 7-18.

Couppié T., Epiphane D. (2001), «Que sont les filles et les garçons devenus? Orientation scolaire atypique et entrée dans la vie active », Céreq-Bref, n 178 .

Couppié T., Epiphane D. (2004a), « Des bancs de l'école aux postes de travail... Chronique d'une ségrégation annoncée », Céreq, Notes Emploi Formation $\mathrm{n}^{\circ} 6$.

Couppié T., Epiphane D. (2004b), «Mesurer et décomposer la ségrégation professionnelle: un travail de composition démesuré ? », in Genre et données longitudinales, sous la direction de A. Degenne, J.-F. Giret, C. Guegnard, J.-J. Paul et P. Werquin, Céreq, Relief $\mathrm{n}^{\circ} 4$.

Couppié T., Epiphane D., Fournier C. (1997), « Insertion professionnelle et début de carrière. Les inégalités entre hommes et femmes résistent-elles au diplôme ? », Céreq-Bref, n 135.

Couppié T., Lopez A. (2003), « Quelle utilité les CAP et BEP tertiaires ont-ils aujourd'hui ? », Céreq-Bref, $\mathrm{n}^{\circ} 196$.

Duncan O.D., Duncan B. (1955) «A methodological Analysis of Segregation Indices », American Sociological Review, $\mathrm{n}^{\circ} 20$, pp. 210-217.

Duru-Bellat M. (1990), L'école des filles. Quelles formations pour quels rôles sociaux?, Paris, L'Harmattan.

Duru-Bellat M., Kieffer A., Marry C. (2001), «La dynamique des scolarités des filles: le double handicap questionné », Revue Française de Sociologie, $\mathrm{n}^{\circ} 42-2$.

Epiphane D., Giret J.-F., Hallier P., Lopez A., Sigot J.C. (2001), « Génération 98 : à qui a profité l'embellie économique ? », Céreq-Bref, ${ }^{\circ} 181$.

Establet R. (1988), « Subversion dans la reproduction scolaire », Revue économique, ${ }^{\circ}$ 1, vol. 39.

Flückiger Y., Silber J. (1999), The Measurement of Segregation in the Labour Force, Physica-Verlag, Heidelberg.

Goux D. et Maurin E. (1997), « Destinées sociales : le rôle de l'école et du milieu d'origine », Économie et Statistique n³06, pp. 13-26.

Karmel T., Maclachlan M. (1988), "Occupational sex segregation. Increasing or decreasing? ”, Economic Record 64 (186), pp. 187-195.

Kessler F. (1997), « Le droit communautaire et l'égalité de traitement entre hommes et femmes: l'approche du juriste », Les Cahiers du Mage, $n^{\circ}$ 3-4.

Maruani M. (2000), Travail et emploi des femmes. Paris, La Découverte, collection « Repères ».

Torsat D. (1999), «Les politiques d'égalité à l'école entre les filles et les garçons, 1984-1999 », in Filles et garçons à l'école: une égalité à construire, sous la direction de Françoise Vouillot, Centre national de documentation pédagogique, collection « Autrement dit».

Silvera R. (1996). Le salaire des femmes, toutes choses inégales?, La Documentation française, Service des Droits des Femmes.

Terrail J.-P. (1992). « Destins scolaires de sexe: une perspective historique et quelques arguments ", Population 47 (3), pp. 645-676.

Vallet L.-A. (1999), «Quarante années de mobilité sociale en France. L'évolution de la fluidité sociale à la lumière de modèles récents ", Revue Française de Sociologie, $\mathrm{n}^{\circ}$ 40-1. 


\section{Résumé}

\section{La ségrégation des hommes et des femmes dans les métiers : entre héritage scolaire et construction sur le marché du travail}

Thomas Couppié et Dominique Epiphane

La distribution des hommes et des femmes dans les différentes professions n'est pas un simple prolongement d'orientations scolaires sexuellement clivées. La ségrégation éducative se convertit - en partie en ségrégation professionnelle au moment de l'entrée dans la vie active. Mais, dans bon nombre de professions, un second mécanisme de ségrégation, indépendant des qualifications scolaires acquises, prend forme sur le marché du travail. Une analyse suivant un processus en deux étapes permet donc de mieux comprendre comment les hommes et les femmes finissent par se trouver plus ou moins séparés dans les différents métiers.

\section{Mots clés}

Division sexuelle du travail, division sexuelle de la formation, statistique, méthodologie, enquête Génération 98

Journal of Economic Literature: J16, C43, J44 